\title{
Vector Higgs-portal dark matter and the invisible Higgs
}

\author{
Oleg Lebedev ${ }^{a},{ }^{*}$ Hyun Min Lee ${ }^{b}{ }^{\dagger}$ and Yann Mambrini ${ }^{c \dagger}$ \\ a DESY Theory Group, Notkestrasse 85, D-22607 Hamburg, Germany \\ ${ }^{b}$ CERN, Theory Division, CH-1211 Geneva 23, Switzerland and \\ ${ }^{c}$ Laboratoire de Physique Théorique Université Paris-Sud, F-91405 Orsay, France
}

\begin{abstract}
The Higgs sector of the Standard Model offers a unique probe of the hidden sector. In this work, we explore the possibility of renormalizable Higgs couplings to the hidden sector vector fields which can constitute dark matter (DM). Abelian gauge sectors with minimal field content, necessary to render the gauge fields massive, have a natural $Z_{2}$ parity. This symmetry ensures stability of the vector fields making them viable dark matter candidates, while evading the usual electroweak constraints. We illustrate this idea with the Stückelberg and Higgs mechanisms. Vector DM is consistent with the WMAP, XENON100, and LHC constraints, while it can affect significantly the invisible Higgs decay. Due to the enhanced branching ratio for the Higgs decay into the longitudinal components of the vector field, the vector Higgs portal provides an efficient way to hide the Higgs at the LHC. This could be the reason why the latest combined ATLAS/CMS data did not bring evidence for the existence of the Higgs boson.
\end{abstract}

\section{INTRODUCTION}

Two of the most important issues in particle physics phenomenology are the nature of dark matter and understanding electroweak symmetry breaking. While $85 \%$ of matter is dark [1], it remains elusive to direct detection and the Weakly Interacting Massive Particle (WIMP) paradigm becomes more and more constrained [2]. On the other front, the accelerator collaborations ATLAS $[3,4]$, CMS [5] and D0/CDF [6, 7] have obtained important results concerning the Higgs searches. If the Higgs boson is the main communicator between the dark world and ours, Higgs hunting is intimately related to direct DM detection. To reconcile all the constraints is then a non-trivial task [8] (see also [9] for more general scenarios). Whereas this idea is normally considered in the context of scalar (or fermion) dark matter, in this work, we study the possibility of vectorial dark matter interacting with the visible sector through the Higgs portal. We show that this framework is well motivated theoretically and, while being consistent with the existing constraints, can affect significantly the ongoing Higgs search at the LHC.

The paper is organized as follows: in section II we introduce the vector Higgs portal and provide its theoretical basis; in section III we study relevant dark matter and accelerator constraints, and discuss implications for the LHC Higgs search; our conclusions are presented in section IV.

\footnotetext{
* lebedev@mail.desy.de

$\dagger$ Hyun.Min.Lee@cern.ch

‡ Yann.Mambrini@th.u-psud.fr
}

\section{VECTOR HIGGS PORTAL}

The Higgs sector offers a unique opportunity to probe the hidden sector. The operator $\bar{H} H$ is the only dim2 gauge and Lorentz invariant operator in the Standard model. Therefore renormalizable interactions of the form

$$
\Delta \mathcal{L}_{\text {scalar }}=\frac{\lambda_{h s}}{4} \bar{H} H\left(S^{2}+m_{h s} S\right)
$$

are possible, where $S$ is a hidden sector (real) scalar. These are known as the scalar "Higgs portal" (the name was coined in [10]). More generally, one can also have a dim-4 vector Higgs portal, which couples the Higgs doublet to a massive vector field $X_{\mu}$ from the hidden sector,

$$
\begin{aligned}
\Delta \mathcal{L}_{\text {vector }} & =\frac{\lambda_{h v}}{4} \bar{H} H X_{\mu} X^{\mu}+\left(\xi_{1} \bar{H} i D_{\mu} H X^{\mu}\right. \\
& \left.+\xi_{2} \bar{H} H i \partial_{\mu} X^{\mu}+\text { h.c. }\right)
\end{aligned}
$$

where the covariant derivative $D_{\mu}$ is taken with respect to the Standard Model (SM) gauge group. ${ }^{1} X_{\mu}$ can be associated with a hidden $\mathrm{U}(1)$. It becomes massive due to the Higgs or Stückelberg mechanism in the hidden sector.

A hidden vector field can be a good dark matter candidate. Suppose its decay into the hidden sector is kinematically forbidden and the only communication with the SM is given by Eq. 2. It is then stable if $\xi_{1,2}$ are zero, in which case the Higgs portal has a $Z_{2}$ symmetry $X_{\mu} \rightarrow-X_{\mu}$. In principle, $X_{\mu}$ can also be made long-lived by adjusting $\xi_{1}$ and $\xi_{2}$ to be very small. In particular, if there are fields charged under the $\mathrm{U}(1)$ apart from those providing longitudinal components of the gauge bosons, these terms are usually generated and it requires non-trivial engineering to make $X_{\mu}$ stable on the cosmological scale.

\footnotetext{
${ }^{1}$ For generality, we have included the $\partial_{\mu} X^{\mu}$ term, which may be relevant in models with field-dependent mass terms.
} 
In the absence of such charged fields, the Lagrangian enjoys a natural $Z_{2}$ parity which makes $X_{\mu}$ stable. This applies both to the Higgs and the Stückelberg mechanisms. Let us consider examples, starting with the less common Stückelberg dark matter.

\section{A. Examples: Stückelberg and "Higgsed" dark matter}

Consider a U(1) vector field which becomes massive through the Stückelberg mechanism (for a review, see [11]),

$$
\mathcal{L}_{\mathrm{St}}=-\frac{1}{4} F_{\mu \nu} F^{\mu \nu}+\frac{1}{2} m^{2} X_{\mu} X^{\mu},
$$

where $X_{\mu}$ is composed of a vector potential $X_{\mu}^{\prime}$ and a Stückelberg axion-like field $\phi$ with the following gauge transformation rule

$$
\begin{aligned}
& X_{\nu} \equiv X_{\nu}^{\prime}+\frac{1}{\mu} \partial_{\nu} \phi, \\
& \delta X_{\nu}^{\prime}=\partial_{\nu} \epsilon, \\
& \delta \phi=-\mu \epsilon .
\end{aligned}
$$

Here $\mu$ is a mass scale and $\epsilon$ is a gauge transfromation parameter. Note that the Lagrangian possesses a symmetry $X_{\mu} \rightarrow-X_{\mu}$, which in terms of the original variables reads $X_{\mu}^{\prime} \rightarrow-X_{\mu}^{\prime}, \phi \rightarrow-\phi .^{2}$ Taking $\mu=m$ corresponds to choosing a canonical kinetic term for the axion. In general, however, $m^{2}$ can be field-dependent, in which case the axion kinetic term involves a function of other fields of the system. Expressing

$$
m^{2}=\mu^{2} f(S),
$$

with $S$ being a scalar, we recover the axion kinetic term $1 / 2 f(S)\left(\partial_{\mu} \phi\right)^{2}$ in the massless limit $\mu \rightarrow 0$. Consider 2 simple possibilities.

(i) One may allow for a Higgs-dependent mass term

$$
m^{2}=\mu^{2}\left(1+\zeta \frac{\bar{H} H}{M^{2}}\right)
$$

where $M$ is another scale. In this case, the Higgs portal coupling $\bar{H} H X_{\mu} X^{\mu}$ is generated with

$$
\lambda_{h v}=2 \zeta \frac{\mu^{2}}{M^{2}} .
$$

A priori neither of these scales is related to the EW scale, nor to each other so that $\lambda_{h v}$ can be significant.

\footnotetext{
2 This symmetry is also preserved by the gauge fixing term.
}

(ii) Consider another example, where $\bar{H} H X_{\mu} X^{\mu}$ is generated through the singlet Higgs portal. Take

$$
m^{2}=\mu^{2} \frac{S^{2}}{M^{2}}
$$

where $S$ is a hidden sector scalar with a potential $V(S)$ and a Higgs portal coupling $\left(\lambda_{h s} / 4\right) \bar{H} H S^{2}$. We have factored out $\mu^{2}$ in order to have a smooth massless limit. Obviously, integrating out heavy $S$ at tree level produces the required coupling with

$$
\lambda_{h v}=2 \lambda_{h s} \frac{\mu^{2}\langle S\rangle^{2}}{M^{2} m_{S}^{2}},
$$

where $m_{S}$ is the mass of $S$. Note that $\lambda_{h v}$ can also be written as $2 \lambda_{h s} m_{X}^{2} / m_{S}^{2}$. The procedure of integrating $S$ out is justified as long as $m_{h}^{2}, m_{X}^{2} \ll m_{S}^{2}$. In practise, these quantities can differ by, say, an order of magnitude so that $\lambda_{h v}$ can be as large as $\mathcal{O}\left(10^{-1}-1\right)$. In the same fashion, self-interaction

$$
\frac{1}{2} \frac{\mu^{4}\langle S\rangle^{2}}{M^{4} m_{S}^{2}}\left(X_{\mu} X^{\mu}\right)^{2}
$$

is also induced. Note also that the physical Higgs boson mixes with $S$ in this example, with the mixing angle being of order $v /\langle S\rangle$.

Furthermore, at one loop, both $\bar{H} H X_{\mu} X^{\mu}$ and $\left(X_{\mu} X^{\mu}\right)^{2}$ are generated with (formally) log-divergent coefficients. Keeping in mind that we are dealing with an effective theory, these are regularized by the cutoff which is expected to be of order $M$. This implies that $\lambda_{h v}$ can be taken as a free parameter at low energies.

As the Higgs doublet develops a VEV, an additional mass term $\lambda_{h v} v^{2} / 2$ for the vector is generated. It can be of either sign, as seen from the above examples. Therefore some cancellation between the original Stückelberg mass and the Higgs-induced one is allowed.

It is important that the linear in $X_{\mu}$ terms vanish in these examples,

$$
\xi_{1}=\xi_{2}=0
$$

This is because, in the absence of fields charged under $\mathrm{U}(1)$ (apart from the axion absorbed in $X_{\mu}$ ), the Lagrangian possesses a natural parity

$$
X_{\mu} \rightarrow-X_{\mu} \text {. }
$$

Note that here we consider a U(1) "orthogonal" to the SM gauge group, so there is no mixing with hypercharge at tree level, nor is it induced radiatively. Thus, the new vector boson evades the usual electroweak constraints.

Some of the ingredients of this construction are well known in string theory. In particular, most of realistic models (see e.g. [12]) involve an "anomalous" U(1) whose anomaly is cancelled by the Green-Schwarz mechanism. In this process, the $\mathrm{U}(1)$ gauge boson becomes massive 
through the Stückelberg mechanism. The corresponding axion has a dilaton-dependent kinetic term, so that the vector mass term in Planck units is (see e.g. [13])

$$
\frac{1}{4 s^{2}}\left(\frac{1}{2} \delta X_{\mu}-\partial_{\mu} a\right)^{2},
$$

where $a$ and $s$ are the axion and the dilaton, respectively, and $\delta$ is a Green-Schwarz parameter. It is therefore quite natural to expect a field-dependent vector boson mass, although in typical string constructions this mass is close to the Planck scale.

(iii) Finally, one can equally well use the Higgs mechanism in the hidden sector. Suppose we have a U(1) gauge field and a charged complex scalar $S$, which has a scalar Higgs portal coupling,

$\mathcal{L}_{\mathrm{Higgs}}=-\frac{1}{4} F_{\mu \nu} F^{\mu \nu}+D_{\mu} S^{*} D^{\mu} S-V(S)+\frac{\lambda_{h s}}{4} \bar{H} H S^{*} S$.

This system enjoys a symmetry (charge conjugation)

$$
\begin{aligned}
X_{\mu}^{\prime} & \rightarrow-X_{\mu}^{\prime}, \\
S & \rightarrow S^{*},
\end{aligned}
$$

where $X_{\mu}^{\prime}$ is the massless gauge field. ${ }^{3}$ This symmetry is retained in the broken phase, that is, the massive gauge field can be reversed: $X_{\mu} \rightarrow-X_{\mu}$. The physical Higgslike excitation has no interactions which are odd under $X_{\mu} \rightarrow-X_{\mu}$, so integrating it out in the unitary gauge will only produce even interactions and $\xi_{1,2}=0$. On the other hand, $\bar{H} H X_{\mu} X^{\mu}$ is generated at both tree and loop level, the latter being log-divergent. Note that the vector field remains light as long as $g^{2} \ll \lambda_{S}$, where $g$ is the gauge coupling and $\lambda_{S}$ is the singlet self-interaction. Otherwise, the analysis is very similar to that of the Stückelberg case.

It is important to remember that these examples are valid within effective field theory. Indeed, we either integrate out a heavy "hidden Higgs" or use higher dimensional operators for the field-dependent kinetic terms. The range of validity of the effective description can be estimated from unitarity considerations. The vector portal coupling together with the $m^{2}$ mass term from the hidden sector results in

$$
\Delta \mathcal{L}_{X}=\frac{1}{2} m_{X}^{2}\left(1+a \frac{2 h}{v}+b \frac{h^{2}}{v^{2}}\right) X_{\mu} X^{\mu},
$$

where the Higgs field is decomposed as $H^{0}=v+h / \sqrt{2}$; $m_{X}^{2}=m^{2}+\frac{1}{2} \lambda_{h v} v^{2}$ and $a / \sqrt{2}=b=\frac{\lambda_{h v} v^{2}}{4 m_{X}^{2}}$. This form is useful for comparison with the Standard Model Higgsvector scattering. The tree level amplitude for the process $h h \rightarrow X X$ (and $h X \rightarrow h X)$ grows with energy as

\footnotetext{
3 Note that this parity does not generalize to the non-abelian case due to the triple gauge boson vertex.
}

$\mathcal{A} \sim\left(a^{2}-b\right) \frac{E^{2}}{v^{2}}$ for $E \gg m_{X}$. Note that in the Standard Model an analogous amplitude vanishes, whereas here it is proportional to the "non-Higgs" mass term $m^{2}$. Requiring the partial wave amplitude $a_{0}$ to be less than $1 / 2$ (see e.g. [14]) and ignoring order 1 factors, we find that the unitarity cutoff is of order

$$
E \sim \sqrt{\frac{16 \pi}{\lambda_{h v}}} \frac{m_{X}^{2}}{m} .
$$

Above this energy, additional states should be taken into account. Further, the amplitude for $X X \rightarrow X X$ proceeding through the Higgs exchange approaches a constant at $E \gg m_{h}, m_{X}$, which imposes $m_{X}>\lambda_{h v} v / \sqrt{8 \pi}$. Finally, in the presence of $X$ self-interaction, unitarity in $X X \rightarrow X X$ requires roughly $E<(8 \pi / \lambda)^{1 / 4} m_{X}$, where $\lambda$ is the $X$ quartic coupling.

We see that there is a fundamental difference between the vector and scalar Higgs portals: the former is UVincomplete, whereas the latter is UV-complete. In the vector case, some amplitudes grow indefinitely with energy meaning that the Higgs portal description breaks down. This can be traced back to the derivative couplings of the Goldstone bosons (or longitudinal polarization vectors growing with energy) and the fact that $m^{2}$ appears as a "hard" mass term. Only in the full theory, which contains extra states, is the "soft" origin of $\mathrm{m}^{2}$ manifest. In contrast, the scalar mass term is not problematic and the scalar Higgs portal is UV-complete.

\section{PHENOMENOLOGY}

The relevant $Z_{2}$-symmetric Lagrangian is given by

$$
\mathcal{L}=\frac{\lambda_{h v}}{4} \bar{H} H X_{\mu} X^{\mu}+\frac{1}{2} m^{2} X_{\mu} X^{\mu}+\frac{\lambda}{4}\left(X_{\mu} X^{\mu}\right)^{2}
$$

Note that upon EW symmetry breaking the mass of the vector field is $m_{X}=\sqrt{m^{2}+\lambda_{h v} v^{2} / 2}$. In what follows, we will take an agnostic attitude to the origin of the various terms and will simply scan over $\lambda_{h v}$ and $m_{X}$ to see their phenomenological implications (see also [15]). We set $\lambda=0$ for simplicity and allow for $\lambda_{h v}$ up to order one, which is consistent with perturbativity up to $10 \mathrm{TeV}$ or so. Since the relevant rates depend on $\lambda_{h v}^{2}$, in the plots we will only display the absolute value of $\lambda_{h v}$, whereas it is understood that its contribution to $m_{X}$ can be of both signs. Finally, reliable computations can be made as long as the unitarity constraint (17) is satisfied. For light $X$, we require the theory to be valid up to the EW scale $\sim v$, whereas for heavy $X$ we take the cutoff to be of order $2 m_{X}$.

In our numerical analysis, we have adapted the code Micromegas [16] to our model in order to compute the thermal WIMP relic abundance. We have also modified the direct detection cross section to take into account recent lattice results on the quark structure of the nucleon, 

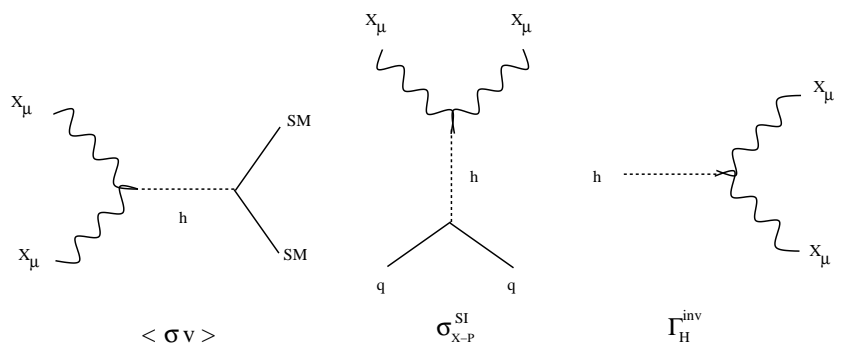

FIG. 1. Feynman diagrams for DM annihilation (left), direct detection (center) and invisible Higgs decay (right).

which appear more reliable than those extracted from the pion-nucleon cross section. Concerning the Higgs physics, we use the code Hdecay [17] to compute the Higgs decay widths.

\section{A. Direct detection and relic abundance constraints}

The Higgs portal coupling allows the vectorial dark matter to annihilate into the light Standard Model species as well as to scatter off nuclei through the Higgs exchange. Fig.1 displays the relevant types of Feynman diagrams. (Numerically, we also take into account annihilation through the quartic vertex $X_{\mu} X^{\mu} h h$ which can be efficient at $m_{X} \gtrsim m_{h}$, although we always find it subdominant.)

Some aspects of the analysis are similar to those of the scalar singlet extension of the SM. The latter was studied in [15, 18-33]. Using this framework, some authors have attempted to explain the DAMA and/or COGENT excess [34-36]. Others have studied its implications for indirect searches [37-39] and constraints from the earlier XENON data [40-42]. In what follows, we analyze the same constraints in the framework of vectorial dark matter. We note that some phenomenological aspects of the vector Higgs portal were studied in [15], while vector dark matter in somewhat different contexts was considered in [43],[44].

The relic abundance of DM is dictated by the $s^{-}$ channel annihilation through the Higgs exchange. For example, the cross section for annihilation into fermions is given by

$$
\left\langle\sigma_{f \bar{f}} v\right\rangle=\frac{\lambda_{h v}^{2} m_{f}^{2}}{48 \pi} \frac{\left(1-m_{f}^{2} / m_{X}^{2}\right)^{3 / 2}}{\left(4 m_{X}^{2}-m_{h}^{2}\right)^{2}} .
$$

The calculational procedure is well known (see e.g. [15]) and here we will only quote the result. The $5 \sigma$ WMAPallowed [1] parameter space $\left\{\lambda_{h v}, m_{X}\right\}$ for $m_{h}=150$ $\mathrm{GeV}$ is shown in Fig. 2 between the two "gull-shaped" curves. As in any Higgs portal model, DM annihilation becomes much more efficient around the Higgs pole,

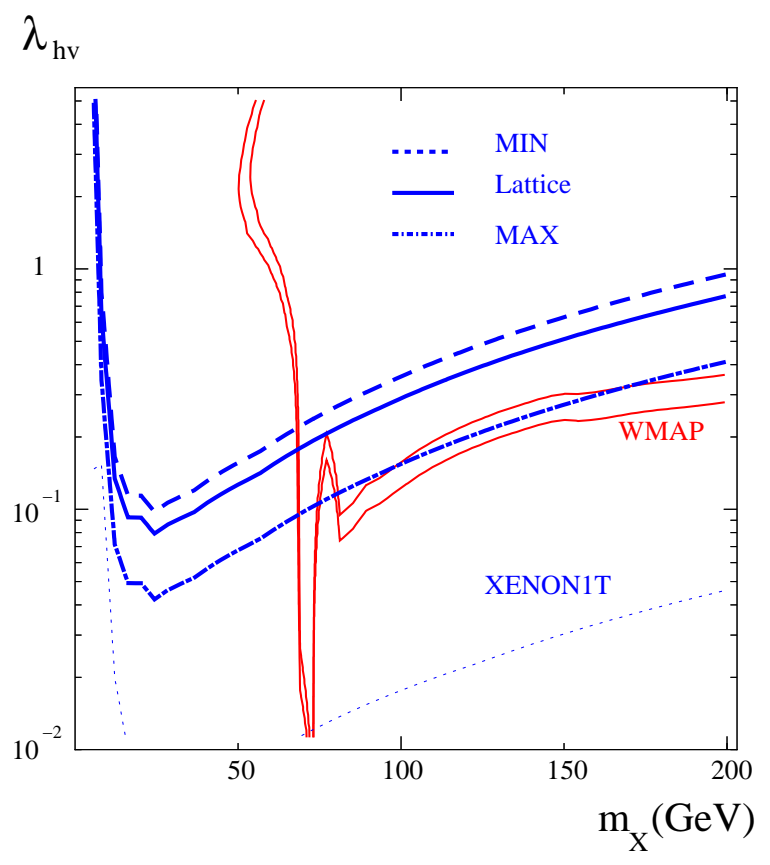

FIG. 2. Parameter space allowed by WMAP (between the "gullshaped" curves) and XENON100 for $m_{h}=150 \mathrm{GeV}$, and prospects for XENON1T.

$m_{h} \simeq 2 m_{X}$, which implies lower values of the coupling $\lambda_{h v}$. In Fig. 2, we also display the XENON100 constraint on the DM-nucleon interaction [2]. The spinindependent cross section is given by

$$
\sigma_{V-N}^{S I}=\frac{\lambda_{h v}^{2}}{16 \pi m_{h}^{4}} \frac{m_{N}^{4} f_{N}^{2}}{\left(m_{X}+m_{N}\right)^{2}}
$$

where $m_{N}$ is the nucleon mass and $f_{N}$ parametrizes the Higgs-nucleon coupling. The latter subsumes contributions of the light quarks $\left(f_{L}\right)$ and heavy quarks $\left(f_{H}\right)$, $f_{N}=\sum f_{L}+3 \times \frac{2}{27} f_{H}$. There exist different estimations of this factor and in what follows we will use the lattice result $f_{N}=0.326[45]$ as well as the MILC results [46] which provide the minimal value $f_{N}=0.260$ and the maximal value $f_{N}=0.629$. Since the cross section is rather sensitive to $f_{N}$, we dispay 3 curves corresponding to these benchmark values. The region above these curves is excluded by XENON100, so the maximal allowed value for $\lambda_{h v}$ at $m_{h}=150 \mathrm{GeV}$ is a few times $10^{-1}$. This is still consistent with WMAP, especially around the resonant annihilation region. We also see that essentially the entire parameter space will be probed by XENON1T. Our results agree well with those of [15] (up to the XENON100 bound which was not available at the time of publication of [15]).

Finally, we have checked that the unitarity constraint (17) is satisfied in the entire WMAP-allowed band, apart from the region $\lambda_{h v}>1$ where DM is light. For $\lambda_{h v} \sim$ $10^{-2}$, the cutoff is almost 2 orders of magnitude above $m_{X}$. 
In what follows, we will use the XENON100 constraint based on the lattice evaluation of $f_{N}$.

\section{B. Implications for the Higgs search}

If the mass of the dark matter particle is less than a half of the Higgs boson mass, dark matter can efficiently be produced in the "invisible" Higgs decay $h \rightarrow X X$. This would affect the Higgs decay branching ratios, while leaving the production cross section unchanged. The partial width for the Higgs decay into vector dark matter can be read off from (16),

$$
\begin{aligned}
\Gamma_{h \rightarrow X_{\mu} X_{\mu}}^{\mathrm{inv}} & =\frac{\lambda_{h v}^{2} v^{2} m_{h}^{3}}{256 \pi m_{X}^{4}}\left(1-4 \frac{m_{X}^{2}}{m_{h}^{2}}+12 \frac{m_{X}^{4}}{m_{h}^{4}}\right) \\
& \times \sqrt{1-4 \frac{m_{X}^{2}}{m_{h}^{2}}}
\end{aligned}
$$

where $v=174 \mathrm{GeV}$. We observe that this width grows fast with the Higgs mass, $\propto m_{h}^{3}$. This is because the decay rate into the longitudinal components of the vector field or, equivalently, the would-be Goldstone bosons, grows with energy due to the derivative couplings of the latter. This is to be contrasted with the decay rate into the usual scalars which decreases with the Higgs mass. Thus even a small coupling $\lambda_{h v}$ can lead to a large invisible branching fraction for a light $X$. In Fig. 3, we show the result of our scan over $\left(m_{h}, m_{X}, \lambda_{h v}\right)$ taking into account the WMAP and XENON100 constraints. We find that points with a large, up to $85 \%$, invisible branching ratio survive for low Higgs masses. There is a sharp fall in $\mathrm{BR}^{\text {inv }}$ around $160 \mathrm{GeV}$, when the $h \rightarrow W W$ mode is in full force. The lightest $m_{X}$ consistent with WMAP and XENON100 is close to $m_{h} / 2$, in which case $\lambda_{h v}$ is smaller than the gauge coupling and the invisible decay is subdominant. Nevertheless, $\mathrm{BR}^{\text {inv }}$ can still be as large as $20-30 \%$.

\section{LHC constraints}

The invisible Higgs decay into dark matter can have a significant impact on the Higgs search at the LHC. Since the standard Higgs decay modes such as $\gamma \gamma, b \bar{b}$, etc. are not affected, the effective branching ratio for a given SM decay $h \rightarrow \alpha \bar{\alpha}$ decreases by a factor of $\mathcal{R}$,

$$
\mathrm{BR}(h \rightarrow \alpha \bar{\alpha})_{\mathrm{SM}+\mathrm{x}_{\mu}}=\mathcal{R} \operatorname{Br}(h \rightarrow \alpha \bar{\alpha})_{\mathrm{SM}},
$$

where

$$
\mathcal{R}=\frac{\Gamma_{\mathrm{SM}}^{\mathrm{tot}}}{\Gamma_{\mathrm{SM}}^{\mathrm{tot}}+\Gamma\left(h \rightarrow X_{\mu} X_{\mu}\right)} .
$$

Since the production modes $q q / g g \rightarrow h$ remain the same, the "measured" cross section for the process $q q / g g \rightarrow$
$\mathrm{Br}(\mathrm{h} \rightarrow \mathrm{XX})$
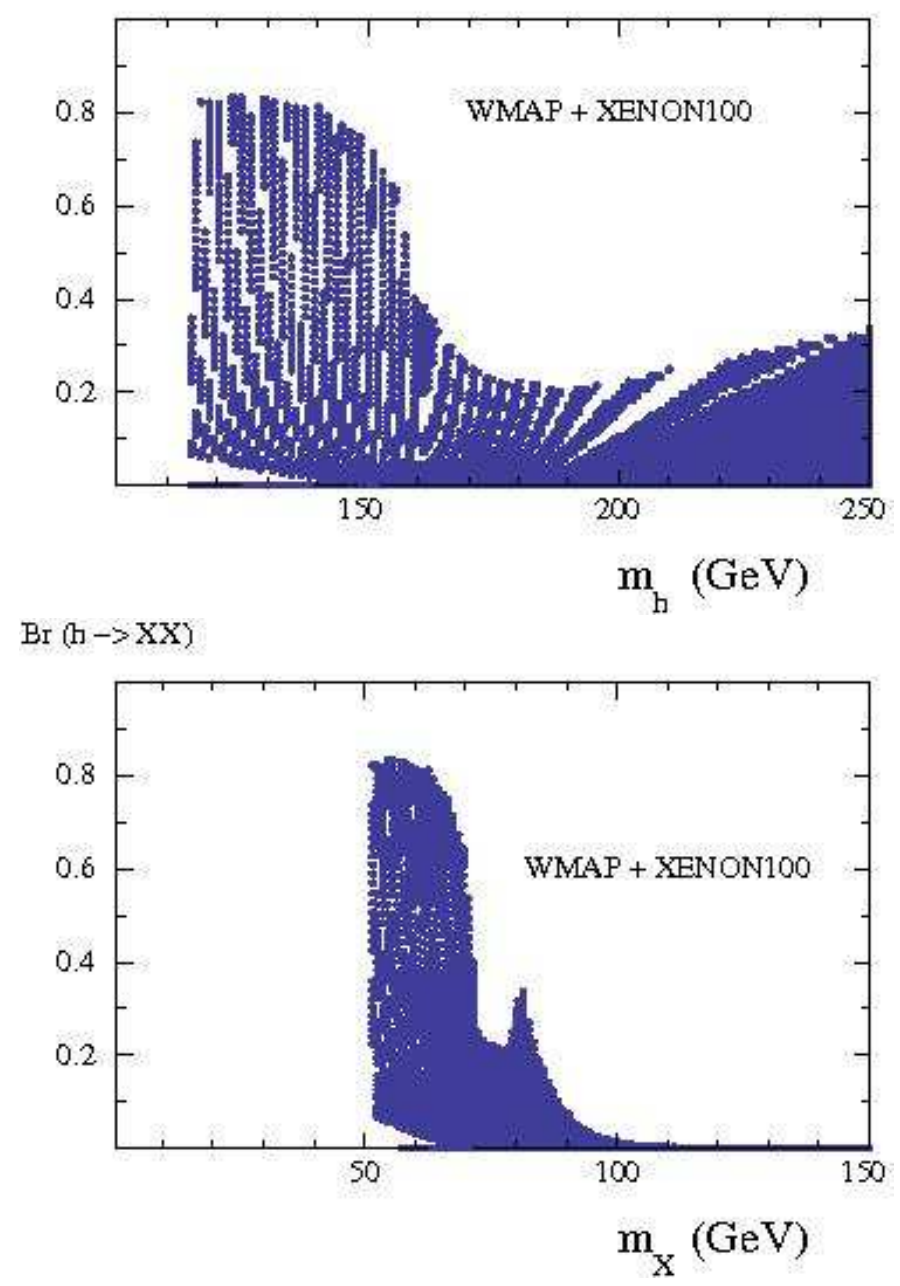

FIG. 3. Branching ratio for the Higgs invisible decay as a function of the Higgs mass (top) and the dark matter mass (bottom). The scan is over $\left(m_{h}, m_{X}, \lambda_{h v}\right)$ and subject to the WMAP and XENON100 constraints.

$h \rightarrow \alpha \bar{\alpha}$ is reduced by a factor of $\mathcal{R}$. Consequently, the event rates used in the ATLAS/CMS analysis [3, 4] would be overestimated by $1 / \mathcal{R}$ and the actual exclusion limits would be weaker. For example, $m_{h} \gtrsim 141 \mathrm{GeV}$ is no longer ruled out by ATLAS/CMS.

Fig. 4 displays the result of our scan over $m_{X}$ and $\lambda_{h v}$ subject to the WMAP, XENON100 and ATLAS/CMS constraints. For each $m_{h}$, we find appropriate $m_{X}$ and $\lambda_{h v}$ such that the WMAP and XENON100 constraints are satisfied and recalculate the cross section for $q q / g g \rightarrow$ $h \rightarrow \alpha \bar{\alpha}$. We find that the invisible decay branching ratio can be as large as $85 \%$ for $m_{h}$ up to $150 \mathrm{GeV}$. For $m_{h}>160 \mathrm{GeV}$ it drops to 20-30\%, in which case the decay is almost SM-like and the corresponding Higgs mass range is largely excluded by ATLAS/CMS. Note, however, that the window $230-250 \mathrm{GeV}$ is still open and consistent with all the constraints. 

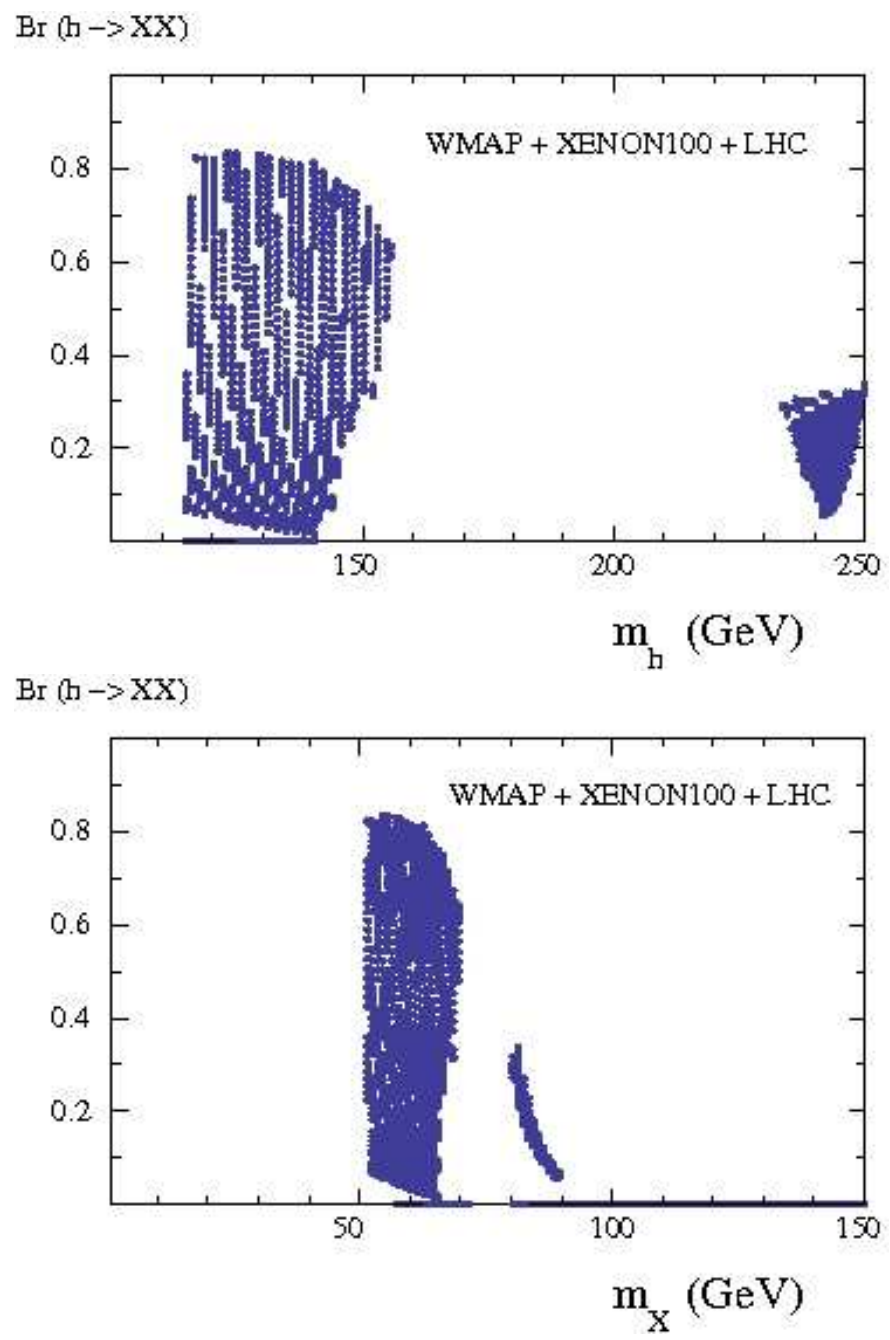

FIG. 4. Branching ratio for the invisible Higgs decay as a function of the Higgs mass (top) and the dark matter mass (bottom). The scan is over $\left(m_{h}, m_{X}, \lambda_{h v}\right)$ and subject to the WMAP, XENON100 and ATLAS/CMS constraints.

The lower bound on $m_{X}$ is determined roughly by $m_{X} \sim m_{h} / 2$ for the minimal possible $m_{h}$, which is about $50 \mathrm{GeV}$. Higher masses $m_{X}>m_{h} / 2$, up to TeV, are allowed by all the constraints. However, the invisible decay is inefficient in this case.

Finally, let us remark that if one gives up the assumption of thermally produced vector dark matter, $\mathrm{BR}^{\text {inv }}$ can be even larger.

\section{Comparison with scalar dark matter}

Analogous analyses have recently appeared in the framework of scalar dark matter interacting with the Standard Model through the Higgs portal [8, 47-50]. Although in many respects the scalar and vector cases are similar, there is an important difference in the invisible decay of the Higgs. While the DM scattering cross section on nucleons is the same in both cases (for the same couplings and masses),

$$
\sigma_{S-N}^{S I}=\frac{\lambda_{h s}^{2}}{16 \pi m_{h}^{4}} \frac{m_{N}^{4} f_{N}^{2}}{\left(m_{S}+m_{N}\right)^{2}},
$$

the invisible Higgs width for scalars is

$$
\Gamma_{h \rightarrow S S}^{\mathrm{inv}}=\frac{\lambda_{h s}^{2} v^{2}}{64 \pi m_{h}} \sqrt{1-4 \frac{m_{S}^{2}}{m_{h}^{2}}},
$$

where $\lambda_{h s}$ is the analog of $\lambda_{h v}$ (see Eq. 1). Unlike in the vector case (21), the decay rate decreases with the Higgs mass. As we mentioned before, this difference has to do with the nature of massive vector fields, which absorb the would-be Goldstone bosons. This results in a higher decay rate at higher energies, i.e. for a heavier Higgs. The decay rate into vectors is enhanced by $m_{h}^{4} / m_{X}^{4}$, which is a large factor for a light $X$. In the region of interest to us, $m_{h} \sim 2 m_{X}$ and the enhancement is an order of magnitude. There are also other numerical differences in the scalar versus vector dark matter. For example, WMAP requires roughly twice as large $\lambda_{h v}$ as $\lambda_{h s}$. This is because the annihilation cross section for scalars is

$$
\left\langle\sigma_{f \bar{f}} v\right\rangle=\frac{\lambda_{h s}^{2} m_{f}^{2}}{16 \pi} \frac{\left(1-m_{f}^{2} / m_{S}^{2}\right)^{3 / 2}}{\left(4 m_{S}^{2}-m_{h}^{2}\right)^{2}},
$$

which is triple that for vectors (due to averaging over polarizations $)^{4}$ with the same couplings and masses. Finally, the phase space coefficients in $\Gamma^{\text {inv }}$ are different for vectors and scalars. We find that these factors compensate each other and the original esimate $\sim m_{h}^{4} / m_{X}^{4}$ gives approximately the right answer. To give an example, at $m_{h}=170 \mathrm{GeV}$ the WMAP/XENON100 constraints are satisfied for $\lambda_{h s}=0.13$ and $m_{S}=71 \mathrm{GeV}$. The corresponding $\mathrm{BR}^{\mathrm{inv}}$ is $2 \%$. The vector counterpart of this example is $\lambda_{h v}=0.21$ and $m_{X}=72 \mathrm{GeV}$, which gives $\mathrm{BR}^{\mathrm{inv}}=25 \%$. As expected, the invisible decay is an order of magnitude more efficient for the vectors.

Although $\Gamma^{\text {inv }}$ for vectors is much larger than that for scalars, the effect on $\mathrm{BR}^{\text {inv }}=\Gamma^{\text {inv }} /\left(\Gamma^{\text {inv }}+\Gamma^{\text {vis }}\right)$ is less significant if the Higgs decays predominantly into dark matter. At $m_{h}<150 \mathrm{GeV}$, the maximal $\mathrm{BR}^{\mathrm{inv}}$ for vectors is $85 \%$ while that for scalars is $65 \%$ (excluding the special case of very light $\sim 5 \mathrm{GeV} \mathrm{DM}$ ). We illustrate this in Fig. 5 which displays $\mathrm{BR}^{\text {inv }}$ consistent with WMAP, XENON100 and ATLAS/CMS constraints for the scalar case. ${ }^{5}$ Note that, up to the above reservations, the Higgs

\footnotetext{
4 At low energies, the vector field can also be viewed as a set of 3 types of scalars which can only annihilate with scalars of the same type. This reduces the annihilation cross section by a factor of 3 .

5 The strip at the top corresponds to very light scalar DM studied in [35].
} 

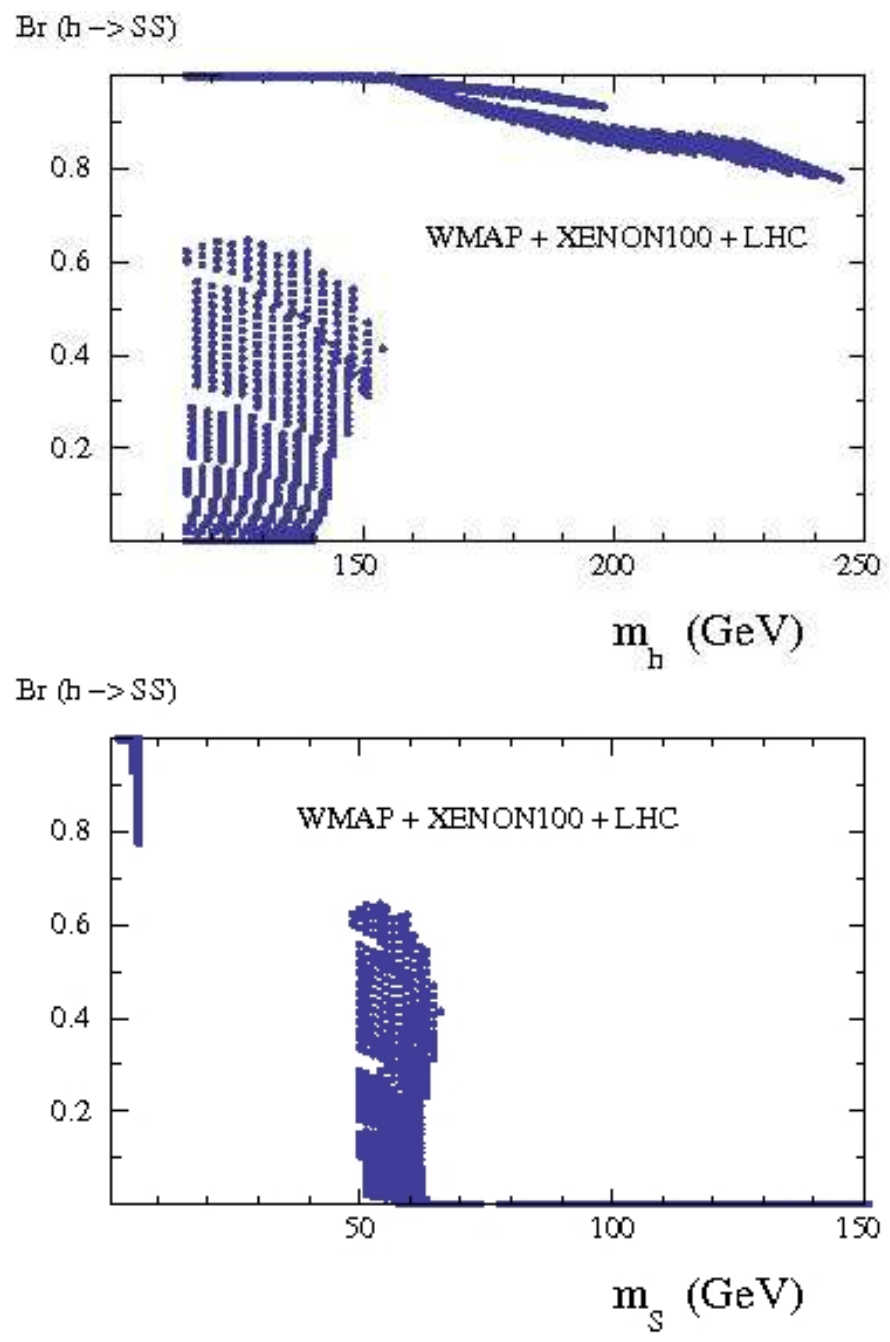

FIG. 5. Branching ratio for the invisible Higgs decay into scalars as a function of the Higgs mass (top) and the dark matter mass (bottom). The scan is over $\left(m_{h}, m_{S}, \lambda_{h s}\right)$ and subject to the WMAP, XENON100 and ATLAS/CMS constraints.

mass window $230-250 \mathrm{GeV}$ is not available since the invisible decay is inefficient for a heavy Higgs.

An important difference between the vector and scalar cases is that in the former case very light dark matter is not allowed by unitarity. For $m_{X} \sim 5 \mathrm{GeV}$ and $\lambda_{h v} \sim 1$, the unitarity cutoff is less $10 \mathrm{GeV}$ meaning that the Higgs portal is not a sensible description of the complete model. Additional states must be invoked to restore unitarity, which in turn would modify our DM analysis. This also applies to the case of $m_{X} \sim 50 \mathrm{GeV}, \lambda_{h v} \sim 2 \mathrm{WMAP}$ allowed region (Fig. 2), where the cutoff is below the EW scale.

\section{CONCLUSION}

We have studied the possibility that dark matter is of vectorial nature and it communicates with the Standard Model through the Higgs portal. We find this possibility well motivated because abelian gauge theories with the minimal field content, which is necessary to render the vector fields massive, possess a natural $Z_{2}$ parity (charge conjugation) making dark matter stable. Vector dark matter is consistent with cosmological and direct detection constraints, and can lead to invisible Higgs decay. The latter is enhanced compared to the scalar dark matter case and provides an efficient way to hide the Higgs at the LHC. A combination of sensitive direct detection experiments (XENON1T) and further collider searches will probe most of parameter space of this scenario.

Acknowledgements. The authors would like to thank particularly M. Kado and J.B. Devivie for their valuable help with the combined ATLAS/CMS analysis. Y.M. thanks E. Bragina and the Magic Monday Journal Club for very useful discussions and comments. We are also grateful to S. Pukhov for his help in solving technical problems related to micrOMEGAs. This work was supported by the French ANR TAPDMS ANR-09-JCJC0146 and the Spanish MICINNs Consolider-Ingenio 2010 Programme under grant Multi- Dark CSD2009-00064. HML is partially supported by the CERN-Korean fellowship.
[1] D. N. Spergel et al. [WMAP Collaboration], "Wilkinson Microwave Anisotropy Probe (WMAP) three year results: Implications for cosmology," Astrophys. J. Suppl. 170 (2007) 377 [arXiv:astro-ph/0603449]; E. Komatsu et al. [WMAP Collaboration], "Five-Year Wilkinson Microwave Anisotropy Probe (WMAP) Observations:Cosmological Interpretation," arXiv:0803.0547 [astro-ph].

[2] E. Aprile et al. [XENON100 Collaboration], arXiv:1104.3121 [astro-ph.CO]; E. Aprile et al. [XENON100 Collaboration], arXiv:1104.2549 [astroph.CO].

[3] report ATLAS-CONF-2011-112

[4] report ATLAS-CONF-2011-157
[5] report CMS PAS HIG-11-011

[6] DO Note 6229-CONF

[7] report FERMILAB-CONF-10-257-E

[8] Y. Mambrini, [arXiv:1108.0671 [hep-ph]]; M. Raidal, A. Strumia, Phys. Rev. D84 (2011) 077701. [arXiv:1108.4903 [hep-ph]]; X. -G. He, J. Tandean, Phys. Rev. D84 (2011) 075018. [arXiv:1109.1277 [hep-ph]].

[9] Y. Mambrini, B. Zaldivar, JCAP 1110 (2011) 023. [arXiv:1106.4819 [hep-ph]].

[10] B. Patt, F. Wilczek, [hep-ph/0605188].

[11] H. Ruegg, M. Ruiz-Altaba, Int. J. Mod. Phys. A19, 32653348 (2004). [arXiv:hep-th/0304245 [hep-th]].

[12] O. Lebedev, H. P. Nilles, S. Ramos-Sanchez, M. Ratz, P. K. S. Vaudrevange, Phys. Lett. B668, 331-335 (2008). 
[arXiv:0807.4384 [hep-th]].

[13] Z. Lalak, S. Lavignac, H. P. Nilles, Nucl. Phys. B559, 48-70 (1999). [hep-th/9903160].

[14] A. Djouadi, Phys. Rept. 457, 1-216 (2008). [hep$\mathrm{ph} / 0503172]$.

[15] S. Kanemura, S. Matsumoto, T. Nabeshima, N. Okada, Phys. Rev. D82 (2010) 055026. [arXiv:1005.5651 [hep$\mathrm{ph}]$.

[16] G. Belanger, F. Boudjema, A. Pukhov and A. Semenov, "micrOMEGAs : a tool for dark matter studies," arXiv:1005.4133 [hep-ph]; G. Belanger, F. Boudjema, A. Pukhov and A. Semenov, "Dark matter direct detection rate in a generic model with micrOMEGAs2.1," Comput. Phys. Commun. 180, 747 (2009) [arXiv:0803.2360 [hep-ph]]; G. Belanger, F. Boudjema, A. Pukhov and A. Semenov, "micrOMEGAs 2.0.7: A program to calculate the relic density of dark matter in a generic model," Comput. Phys. Commun. 177, 894 (2007).

[17] A. Djouadi, J. Kalinowski, M. Spira, Comput. Phys. Commun. 108 (1998) 56-74. [hep-ph/9704448].

[18] V. Silveira, A. Zee, Phys. Lett. B161, 136 (1985).

[19] In the context of mirror matter, the Higgs portal interaction also appeared in R. Foot, H. Lew and R. R. Volkas, Phys. Lett. B 272, 67 (1991).

[20] J. McDonald, Phys. Rev. D50 (1994) 3637-3649. [hepph/0702143 [hep-ph]].

[21] J. McDonald, Phys. Rev. Lett. 88 (2002) 091304. [hep$\mathrm{ph} / 0106249]$.

[22] C. P. Burgess, M. Pospelov, T. ter Veldhuis, Nucl. Phys. B619 (2001) 709-728. [hep-ph/0011335].

[23] K. A. Meissner, H. Nicolai, Phys. Lett. B648 (2007) 312-317. [hep-th/0612165].

[24] H. Davoudiasl, R. Kitano, T. Li, H. Murayama, Phys. Lett. B609 (2005) 117-123. [hep-ph/0405097].

[25] X. -G. He, T. Li, X. -Q. Li, J. Tandean, H. -C. Tsai, Phys. Rev. D79 (2009) 023521. [arXiv:0811.0658 [hepph]]; X. -G. He, T. Li, X. -Q. Li, J. Tandean, H. -C. Tsai, Phys. Lett. B688 (2010) 332-336. [arXiv:0912.4722 [hepph]]; X. -G. He, S. -Y. Ho, J. Tandean, H. -C. Tsai, Phys. Rev. D82 (2010) 035016. [arXiv:1004.3464 [hep-ph]].

[26] M. Aoki, S. Kanemura, O. Seto, Phys. Lett. B685 (2010) 313-317. [arXiv:0912.5536 [hep-ph]].

[27] W. -L. Guo, Y. -L. Wu, JHEP $1010 \quad$ (2010) 083. [arXiv:1006.2518 [hep-ph]].

[28] V. Barger, Y. Gao, M. McCaskey, G. Shaughnessy, Phys. Rev. D82 (2010) 095011. [arXiv:1008.1796 [hep-ph]].

[29] J. R. Espinosa, M. Quiros, Phys. Rev. D76 (2007) 076004. [hep-ph/0701145].

[30] H. Sung Cheon, S. K. Kang, C. S. Kim, JCAP 0805
(2008) 004. [arXiv:0710.2416 [hep-ph]].

[31] V. Barger, P. Langacker, M. McCaskey, M. J. RamseyMusolf, G. Shaughnessy, Phys. Rev. D77 (2008) 035005. [arXiv:0706.4311 [hep-ph]].

[32] T. E. Clark, B. Liu, S. T. Love, T. ter Veldhuis, Phys. Rev. D80, 075019 (2009), [arXiv:0906.5595 [hepph]]; R. N. Lerner, J. McDonald, Phys. Rev. D80, 123507 (2009), [arXiv:0909.0520 [hep-ph]]; O. Lebedev, H. M. Lee, [arXiv:1105.2284 [hep-ph]].

[33] C. Englert, T. Plehn, D. Zerwas and P. M. Zerwas, Phys. Lett. B 703, 298 (2011) [arXiv:1106.3097 [hep-ph]].

[34] S. Andreas, T. Hambye, M. H. G. Tytgat, JCAP 0810 (2008) 034. [arXiv:0808.0255 [hep-ph]].

[35] S. Andreas, C. Arina, T. Hambye, F. -S. Ling, M. H. G. Tytgat, Phys. Rev. D82 (2010) 043522. [arXiv:1003.2595 [hep-ph]].

[36] M. H. G. Tytgat, [arXiv:1012.0576 [hep-ph]].

[37] C. E. Yaguna, JCAP 0903 (2009) 003. [arXiv:0810.4267 [hep-ph]].

[38] A. Goudelis, Y. Mambrini, C. Yaguna, JCAP 0912 (2009) 008. [arXiv:0909.2799 [hep-ph]].

[39] C. Arina, M. H. G. Tytgat, JCAP 1101 (2011) 011. [arXiv:1007.2765 [astro-ph.CO]].

[40] Y. Cai, X. -G. He, B. Ren, Phys. Rev. D83 (2011) 083524. [arXiv:1102.1522 [hep-ph]].

[41] A. Biswas, D. Majumdar, [arXiv:1102.3024 [hep-ph]].

[42] M. Farina, M. Kadastik, D. Pappadopulo, J. Pata, M. Raidal, A. Strumia, [arXiv:1104.3572 [hep-ph]].

[43] T. Hambye, JHEP 0901, 028 (2009) [arXiv:0811.0172 [hep-ph]]; T. Hambye and M. H. G. Tytgat, Phys. Lett. B 683, 39 (2010) [arXiv:0907.1007 [hep-ph]].

[44] J. Hisano, K. Ishiwata, N. Nagata and M. Yamanaka, Prog. Theor. Phys. 126, 435 (2011) [arXiv:1012.5455 [hep-ph]].

[45] R. D. Young, A. W. Thomas, Phys. Rev. D81 (2010) 014503. [arXiv:0901.3310 [hep-lat]].

[46] D. Toussaint et al. [ MILC Collaboration ], Phys. Rev. Lett. 103 (2009) 122002. [arXiv:0905.2432 [hep-lat]].

[47] M. C. Bento, O. Bertolami, R. Rosenfeld, Phys. Lett. B518 (2001) 276-281 [hep-ph/0103340]; M. C. Bento, O. Bertolami, R. Rosenfeld, L. Teodoro, Phys. Rev. D62 (2000) 041302 [astro-ph/0003350].

[48] M. Gonderinger, Y. Li, H. Patel, M. J. Ramsey-Musolf, JHEP 1001 (2010) 053. [arXiv:0910.3167 [hep-ph]].

[49] I. Low, P. Schwaller, G. Shaughnessy, C. E. M. Wagner, [arXiv:1110.4405 [hep-ph]]; M. Pospelov, A. Ritz, [arXiv:1109.4872 [hep-ph]].

[50] R. Foot, A. Kobakhidze, R. R. Volkas, [arXiv:1109.0919 [hep-ph]]; E. Weihs, J. Zurita, [arXiv:1110.5909 [hep-ph]]; P. J. Fox, R. Harnik, J. Kopp, Y. Tsai, [arXiv:1109.4398 [hep-ph]]. 